\title{
Damià Barceló (Ed.): Fuel oxygenates
}

\author{
L. Reijnders
}

Published online: 2 September 2008

(C) The Author(s) 2008. This article is published with open access at Springerlink.com

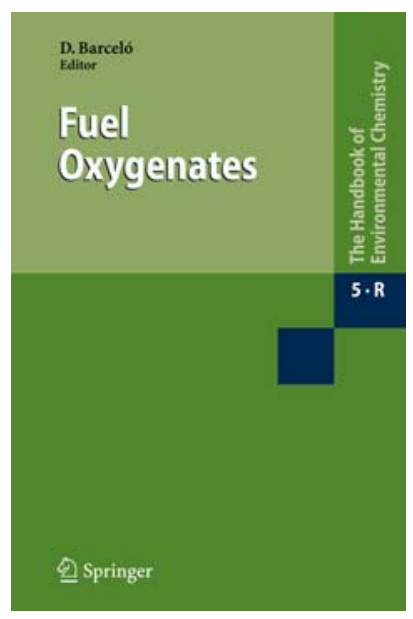

Book's topic Mainly to improve air quality, the fuel oxygenate methyl tert-butyl ether (MTBE) became an important ingredient of gasoline. Its success in this respect was however followed by a fall from environmental grace, especially in the U.S.A., due its widespread emergence as a contaminant of groundwater. MTBE is now rapidly replaced by other oxygenates such as ETBE (ethyl tertbutyl ether). Fuel Oxygenates reviews the toxicity of fuel oxygenates, analytical methods for their determination in water, the occurrence and fate of MTBE in the aquatic environment and remediation of water contaminated by fuel oxygenates, especially MTBE.

Contents There are 14 contributions to Fuel Oxygenates. Two deal with oxygenate toxicity and three with analytical methods and the occurrence of oxygenates and their key

\footnotetext{
L. Reijnders $(\square)$

Institute of Biodiversity and Ecosystem Dynamics,

University of Amsterdam,

Nieuwe Achtergracht 166,

1018 VW Amsterdam, The Netherlands

e-mail: 1.reijnders@uva.nl
}

degradation intermediates. The other chapters are focused on remediation, mostly on the remediation of MTBE present in groundwater. One of the latter contributions has an analytical focus as it deals with compound specific isotope analysis, an analytical technique that has drawn attention because of it alleged ability to quantify degradation rates. In the contributions about remediation, both biotic and a biotic remediation options are considered.

Comparison with existing literature There have been several reviews in scientific journals dealing with the aspects of fuel oxygenates in the aquatic environment which are covered in this book. However those reviews have been published in a wide variety of journals, and it is quite useful to have one book reviewing all the aspects. Nevertheless an avid reader of reviews in scientific journals may find texts that are already very familiar. For instance, the toxicological review of MTBE and ETBE by Douglas McGregor largely overlaps the reviews that he wrote for the Critical Reviews in Toxicology.

Critical assessment Much of the reviewing in Fuel Oxygenates is competent. Duplication is limited and there are only a few remarkable lapses, such as the sentence on page 121 which states that the longest plume of chlorinated aliphatic hydrocarbons recorded in literature so far amounts to $10000 \mathrm{~km}$, which should rather be $10000 \mathrm{~m}$. A very good contribution is Removal of MTBE and other fuel oxygenates during drinking water treatment of Christine Baus and Hein-Jürgen Brauch. They make clear that removal of fuel oxygenates without unwanted side effects is far from easy and entails considerable cost. However, Fuel Oxygenates could have been a better book. It would have benefited from a chapter explicitly dealing with the question whether or not ETBE will go the same way as MTBE. Secondly Fuel Oxygenates could have better handled the remarkable fall from grace of MTBE. If one reads Fuel Oxygenates one wonders why the fuss, because 
it would seem, after reading the contributions of John Fawell and Monica Rossel, Silvia Lacorte and Dami Barceló, that odor is the only reason for cleaning up wells. In practice however the demise of MTBE is strongly linked to a public debate about experiments with rodents showing its carcinogenicity. The International Agency for Research on Cancer (IARC) has classified MTBE on this basis as "not classifiable as to its carcinogenicity to humans, based on limited evidence in experimental animals and inadequate evidence in humans'. Whereas some have interpreted this to mean that it is not necessary to develop a health-based standard or guideline for MTBE, others have defended the position that in absence of sufficient data one should rather err on the safe side as MTBE is a potential carcinogen.

Readership recommendation This book is recommended for scientists dealing with remediation of water contaminated by fuel oxygenates.

Open Access This article is distributed under the terms of the Creative Commons Attribution Noncommercial License which permits any noncommercial use, distribution, and reproduction in any medium, provided the original author(s) and source are credited. 